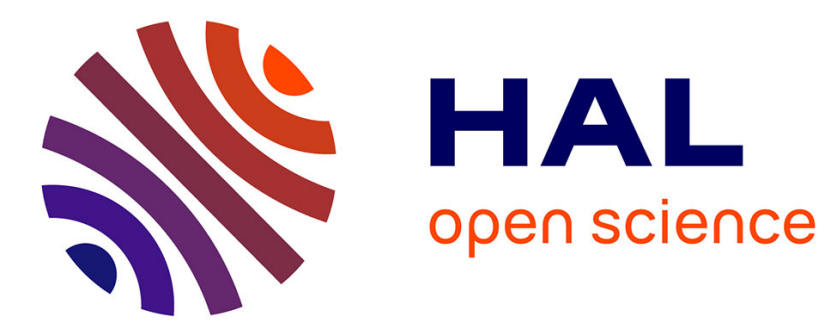

\title{
Etude et description d'un nouveau thermofluxmètre permettant la mesure simultanée des flux thermiques et des variations de température
}

\author{
G. Ravalitera, M. Cornet, B. Duthoit, P. Thery
}

\section{- To cite this version:}

G. Ravalitera, M. Cornet, B. Duthoit, P. Thery. Etude et description d'un nouveau thermofluxmètre permettant la mesure simultanée des flux thermiques et des variations de température. Revue de Physique Appliquée, 1982, 17 (4), pp.177-185. 10.1051/rphysap:01982001704017700 . jpa-00244987

\section{HAL Id: jpa-00244987 https://hal.science/jpa-00244987}

Submitted on 1 Jan 1982

HAL is a multi-disciplinary open access archive for the deposit and dissemination of scientific research documents, whether they are published or not. The documents may come from teaching and research institutions in France or abroad, or from public or private research centers.
L'archive ouverte pluridisciplinaire HAL, est destinée au dépôt et à la diffusion de documents scientifiques de niveau recherche, publiés ou non, émanant des établissements d'enseignement et de recherche français ou étrangers, des laboratoires publics ou privés. 
Classification

Physics Abstracts

$72.40 \mathrm{P}-07.20 \mathrm{~F}$

\title{
Etude et description d'un nouveau thermofluxmètre permettant la mesure simultanée des flux thermiques et des variations de température
}

\author{
G. Ravalitera $\left({ }^{*}\right)$, M. Cornet $\left({ }^{*}\right)$, B. Duthoit et P. Thery $\left({ }^{*}\right)$ \\ (*) CRESMAT, Centre de Recherche Science des Matériaux et Techniques de Construction, Bât. P3, $3^{e}$ étage, \\ Université de Lille-1, 59655 Villeneuve d'Ascq Cedex, France
}

(Reçu le 15 juillet 1981, révisé le 14 octobre 1981, accepté le 20 octobre 1981)

\begin{abstract}
Résumé. - Les résultats concernant l'effet thermoélectrique dans les feuilles minces recouvertes d'un dépôt électrolytique ont permis la réalisation de plusieurs types de cellules fluxmétriques.

Une thermopile de conception nouvelle a été réalisée en intégrant un grand nombre de thermoéléments sur une feuille conductrice de faible épaisseur. La tension apparaissant aux bornes du circuit thermoélectrique est proportionnelle au flux thermique traversant la surface de mesure. La résistance interne varie avec la température. La mesure de la résistance interne fournit une information électrique représentative de la température du capteur. Le capteur présente l'avantage d'être réalisable de façon industrielle par mise en œuvre de la technologie des circuits imprimés.
\end{abstract}

\begin{abstract}
Preceding results concerning thermoelectric effect in thin conductor sheets covered with an electrolytic deposit have been used to realize new type of heat flow cells. New types of thermopile have been developped by integrating a lot of thermoelements on a thin conductor sheet. The e.m.f. measured on thermoelectric circuit is proportionnal to the heat flow crossing the measure aera. Internal resistance depends upon temperature. Internal resistance measurement give an electrical information representative of sensor temperature. The sensor can be manufactured by using technology of printed circuit.
\end{abstract}

Introduction. - Depuis quelques années, les programmes d'économie d'énergie posent de nouveaux problèmes de mesure dans le domaine de la thermique. La gestion des échanges de chaleur nécessite la mesure simultanée des quantités de chaleur échangée et du niveau de température auquel s'effectuent les échanges. La nécessité de réaliser des fluxmètres thermiques réactualise l'intérêt des recherches en thermoélectricité. Notre travail se situe dans ce contexte et décrit un capteur permettant la mesure simultanée d'un flux thermique et de la température moyenne de la surface de mesure du capteur.

La réalisation du capteur a nécessité plusieurs innovations scientifiques et technologiques :

- La mise au point du thermocouple de surface réalisé en déposant par voie électrolytique une couche métallique sur une feuille conductrice de nature différente.

- La mise au point de cellules fluxmétriques réalisées à partir de thermocouples de surface. Elles sont conçues de façon à générer une information électrique proportionnelle au flux thermique à mesurer.

- La mise au point d'une technologie à intégration de thermocouples. Les cellules fluxmétriques sont de dimensions inférieures au millimètre et peuvent être intégrées en grand nombre sur la surface de mesure. Les techniques de circuits imprimés peuvent être utilisées pour réaliser les thermofluxmètres. Avec un équipement classique, nous intégrons. actuellement une cinquantaine de thermoéléments par centimètre carré.

Pour réaliser un fluxmètre thermique, il faut successivement résoudre deux problèmes. Le premier est un problème de thermoélectricité qui consiste à établir une relation entre les grandeurs électrique et thermique. Le second est un problème de thermique qui consiste à établir une relation entre le flux thermique à mesurer et la grandeur thermique effectivement mesurée par le thermocouple de surface. Le schéma électrique équivalent du capteur est constitué par un générateur de tension idéal de f.é.m. proportionnelle au flux à mesurer en série avec une résistance interne de valeur variable avec la température. La mesure simultanée de la tension à vide et de la résistance du circuit électrique a permis de mesurer simultanément le flux de chaleur et la température de la surface de mesure.

Disposant d'informations électriques proportionnelles aux flux et variations de température, la mise en ouvre de méthodes de traitement classiques 
dans le domaine de l'électronique permet d'obtenir des informations numériques représentatives des grandeurs à mesurer.

Ces informations peuvent être traitées par des systèmes à microprocesseurs. Cette instrumentation permet d'utiliser le fluxmètre dans les problèmes de gestion et de contrôle des échanges thermiques.

1. Caractéristique électrique d'un thermocouple de surface. - La figure 1-a représente un thermocouple de surface réalisé en déposant une couche électrolytique de cuivre sur une feuille de constantan. Un tel dispositif a des propriétés thermoélectriques particulières qui peuvent être représentées en première approximation en déterminant les effets électriques induits par la composante tangentielle $\nabla T$ d'un gradient thermique imposé sur la surface du thermoélément. Les champs électromoteurs induits par effet thermoélectrique dans chacun des conducteurs ont des valeurs différentes. Il en résulte un déséquilibre électrique et l'apparition de courants induits distribués sur la surface du thermoélément. Les chutes ohmiques provoquées par la circulation de ces courants génèrent une tension à vide dont l'expression a été calculée dans un article précédent [1]. Le développement présenté ci-dessous complète l'étude précédente en tenant compte des interactions localisées sur les frontières du dépôt électrolytique. Il permet également de calculer le courant de sortie du thermoélément fermé sur une charge quelconque puisque nous établissons le schéma électrique équivalent au thermocouple.

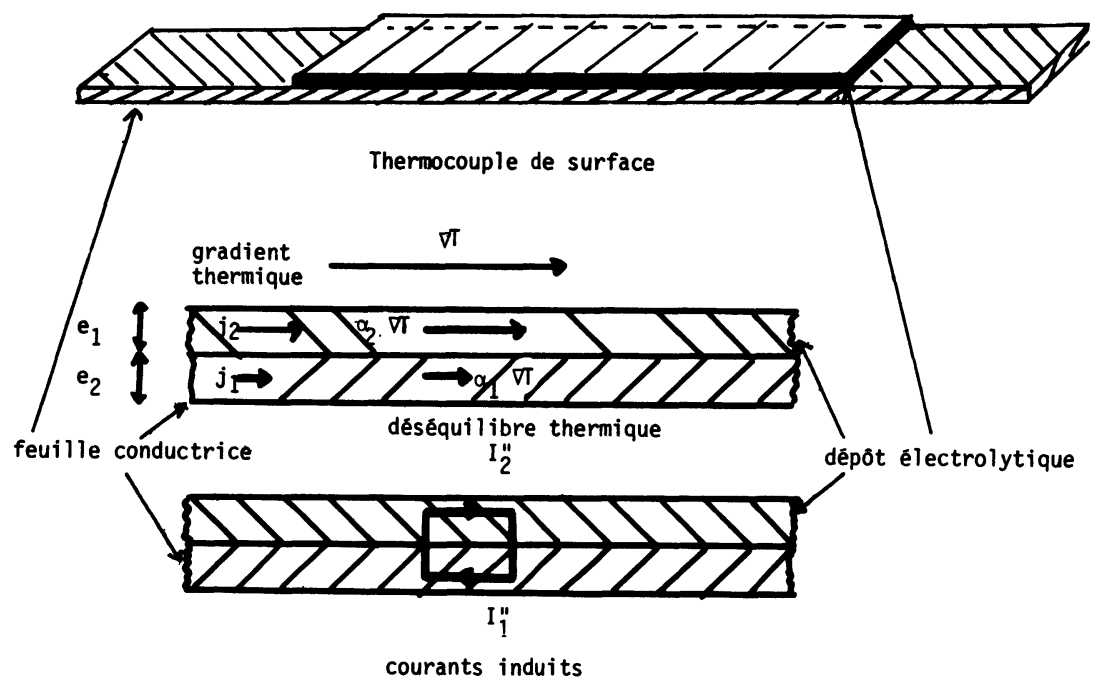

Fig. 1. - Représentation du déséquilibre électrique dans un système à deux conducteurs superposés soumis au gradient thermique $\nabla T: 1 a)$ Représentation des champs électromoteurs $\alpha_{1} \nabla T$ et $\alpha_{2} \nabla T ; 1 b$ ) Courants induits par le déséquilibre électrique local.

[Electrical disequilibrium induced by a tangential thermal gradient in a thin electroplated sheet : $1 a$ ) Thermoelectrical fields $\left.\alpha_{1} \frac{\partial T}{\partial x}, \alpha_{2} \frac{\partial T}{\partial x} ; 1 b\right)$ Induced currents by the thermal gradient. ]

1.1 EFFETS THERMOÉLECTRIQUES DISTRIBUÉS SUR LA SURFACE DU THERMOCOUPLE. - Les densités de courant électrique $j$ et de flux thermique $\phi$ dans un conducteur monodimensionnel soumis simultanément à un champ électrique $E$ et à un gradient thermique $\partial T / \partial x$ sont représentées par les équations phénoménologiques [1]

$$
\begin{aligned}
& j=\sigma E-\alpha \sigma \nabla T \\
& \phi=\alpha T j-\lambda \nabla T
\end{aligned}
$$

$\sigma$ conductivité électrique isotherme

$\lambda$ conductivitè thermique à courant électrique constant

$\alpha$ pouvoir thermoélectrique

$T$ température absolue.
La première équation permet de déterminer les densités de courant électrique dans le thermocouple de surface à deux conducteurs superposés représenté figure 1. La valeur locale du gradient thermique tangentiel étant $\nabla T$, les densités de courant $j_{1}$ et $j_{2}$ sont définies par les relations :

$$
\begin{aligned}
& j_{1}=\sigma_{1} E_{1}-\alpha_{1} \sigma_{1} \nabla T \\
& j_{2}=\sigma_{2} E_{2}-\alpha_{2} \sigma_{2} \nabla T
\end{aligned}
$$

De part et d'autre de la surface de séparation des conducteurs superposés, il y a égalité des composantes tangentielles du champ électrique

$$
E_{1}(x)=E_{2}(x)=E(x) .
$$


D'autre part pour un circuit de largeur unité, les courants dans chacune des couches conductrices ont pour valeurs :

$$
I_{1}=e_{1} j_{1} \quad I_{2}=e_{2} j_{2} .
$$

Soit, compte tenu des relations précédentes :

$$
\begin{aligned}
& I_{1}=\sigma_{1} e_{1} E-\alpha_{1} \sigma_{1} e_{1} \nabla T . \\
& I_{2}=\sigma_{2} e_{2} E-\alpha_{2} \sigma_{2} e_{2} \nabla T .
\end{aligned}
$$

En régime quasi stationnaire, l'intensité électrique $I$ a une valeur uniforme dans tout le circuit :

$$
I=I_{1}+I_{2} \text {. }
$$

D'où l'expression de la caractéristique :

$$
I=\left(\sigma_{1} e_{1}+\sigma_{2} e_{2}\right) E-\left(\alpha_{1} \sigma_{1} e_{1}+\alpha_{2} \sigma_{2} e_{2}\right) \nabla T .
$$

A partir des relations (9), (7) et (8), nous obtenons trois expressions distinctes pour le champ électrique :

$$
\begin{gathered}
E=\frac{\left(\alpha_{1} \sigma_{1} e_{1}+\alpha_{2} \sigma_{2} e_{2}\right)}{\left(\sigma_{1} e_{1}+\sigma_{2} e_{2}\right)} \nabla T+\frac{I}{\sigma_{1} e_{1}+\sigma_{2} e_{2}} \\
E=\alpha_{1} \nabla T+\frac{I_{1}}{\sigma_{1} e_{1}} \\
E=\alpha_{2} \nabla T+\frac{I_{2}}{\sigma_{2} e_{2}}
\end{gathered}
$$

Dans ces équations les variables thermiques et électriques sont indépendantes l'une de l'autre. Les relations précédentes ne peuvent être vérifiées pour une valeur unique de $E$ que si les courants $I_{1}$ et $I_{2}$ ont chacun une composante proportionnelle à $\nabla T$ permettant de compenser l'inégalité $\alpha_{1} \neq \alpha_{2}$. Pour déterminer ces composantes $I_{1}^{\prime \prime}$ et $I_{2}^{\prime \prime}$ proportionnelles à $\nabla T$, il suffit de remplacer dans les équations (10) et (11) le champ électrique par sa valeur tirée de l'équation (12). Les courants $I_{1}$ et $I_{2}$ sont alors représentés par les équations :

$$
\begin{aligned}
I_{1} & =\frac{\sigma_{1} e_{1} I}{\sigma_{1} e_{1}+\sigma_{2} e_{2}}+ \\
& +\frac{\sigma_{1} \sigma_{2} e_{1} e_{2}}{\left(\sigma_{1} e_{1}+\sigma_{2} e_{2}\right)}\left(\alpha_{2}-\alpha_{1}\right) \nabla T=I_{1}^{\prime}+I_{1}^{\prime \prime} \\
I_{2} & =\frac{\sigma_{2} e_{2} I}{\sigma_{1} e_{1}+\sigma_{2} e_{2}}- \\
& -\frac{\sigma_{1} \sigma_{2} e_{1} e_{2}}{\left(\sigma_{1} e_{1}+\sigma_{2} e_{2}\right)}\left(\alpha_{2}-\alpha_{1}\right) \nabla T=I_{2}^{\prime}+I_{2}^{\prime \prime} .
\end{aligned}
$$

Les courants $I_{1}$ et $I_{2}$ s'expriment par la superposition de composantes purement électriques $I_{1}^{\prime}$ et $I_{2}^{\prime}$ dans le rapport des conductances électriques

$$
I_{1}^{\prime}=\frac{\sigma_{1} e_{1}}{\sigma_{1} e_{1}+\sigma_{2} e_{2}} I \quad I_{2}^{\prime}=\frac{\sigma_{2} e_{2}}{\sigma_{1} e_{1}+\sigma_{2} e_{2}} I
$$

et de composantes thermoélectriques

$$
\begin{aligned}
& I_{1}^{\prime \prime}=\frac{\sigma_{1} \sigma_{2} e_{1} e_{2}}{\left(\sigma_{1} e_{1}+\sigma_{2} e_{2}\right)}\left(\alpha_{2}-\alpha_{1}\right) \nabla T \\
& I_{2}^{\prime \prime}=-\frac{\sigma_{1} \sigma_{2} e_{1} e_{2}}{\left(\sigma_{1} e_{1}+\sigma_{2} e_{2}\right)} \nabla T\left(\alpha_{2}-\alpha_{1}\right) .
\end{aligned}
$$

Les courants $I_{1}^{\prime \prime}$ et $I_{2}^{\prime \prime}$ permettent de vérifier la condition de passage (5) quel que soit le courant électrique circulant dans le circuit. Les courants $I_{1}^{\prime \prime}$ et $I_{2}^{\prime \prime}$ représentent des échanges thermiques d'origine thermoélectrique qui peuvent être traités indépendamment des effets irréversibles dus à la circulation des courants $I_{1}^{\prime}$ et $I_{2}^{\prime}$. Quel que soit l'état du système, la relation :

$$
I_{1}^{\prime \prime}+I_{2}^{\prime \prime}=0
$$

est vérifiée en chaque point de la surface d'échange.

On peut considérer que les courants $I_{1}^{\prime \prime}$ et $I_{2}^{\prime \prime}$ induits par effet thermoélectrique se referment en chaque point à travers la surface de séparation des conducteurs superposés. Tout se passe comme si le système était constitué de thermocouples superficiels en courtcircuit de f.é.m. à vide $\left(\alpha_{2}-\alpha_{1}\right) \nabla T$ par unité de longueur débitant dans une conductance égale à $\sigma_{1} \sigma_{2} e_{1} e_{2} / \sigma_{1} e_{1}+\sigma_{1} e_{2}$ par unité de longueur. Comme dans les couples classiques, les tensions sont induites par effets Thomson et Peltier. Les valeurs des courants $I_{1}^{\prime \prime}$ et $I_{2}^{\prime \prime}$ dans chacune des feuilles conductrices superposées sont telles que les chutes ohmiques provoquées par le passage de ces courants compensent localement le déséquilibre dû à l'inégalité des champs thermoélectriques $\alpha_{1} \nabla T$ et $\alpha_{2} \nabla T$. Il est en effet facile de vérifier les relations :

$$
\begin{aligned}
& E=\alpha_{1} \nabla T+\frac{I_{1}^{\prime \prime}}{\sigma_{1} e_{1}}+\frac{I}{\sigma_{1} e_{1}+\sigma_{2} e_{2}} \\
& E=\alpha_{2} \nabla T+\frac{I_{2}^{\prime \prime}}{\sigma_{2} e_{2}}+\frac{I}{\sigma_{1} e_{1}+\sigma_{2} e_{2}} .
\end{aligned}
$$

Les équations (10) et (16) représentent le champ électrique dans les connexions et dans la surface d'échange du thermocouple de surface représenté figure 2. La tension apparaissant aux bornes des connexions de sortie s'exprime en fonction du courant électrique $I$ traversant le circuit et de la différence de température imposée sur la surface d'échange :

$$
\Delta V=\int_{\substack{\mathrm{c} \\ \text { connexions }}} E \mathrm{~d} l+\int_{\substack{\mathrm{s} \\ \text { surface d'echange }}} E \mathrm{~d} l
$$




$$
\begin{aligned}
\Delta V=\int_{\mathrm{c}} & \left(\alpha_{1} \nabla T+\frac{I}{\sigma_{1} e_{1}}\right) \mathrm{d} l+ \\
& +\int_{\mathrm{s}}\left|\alpha_{1} \nabla T+\frac{I_{1}^{\prime \prime}}{\left(\sigma_{1} e_{1}\right)}+\frac{I}{\sigma_{1} e_{1}+\sigma_{2} e_{2}}\right| \mathrm{d} l .
\end{aligned}
$$
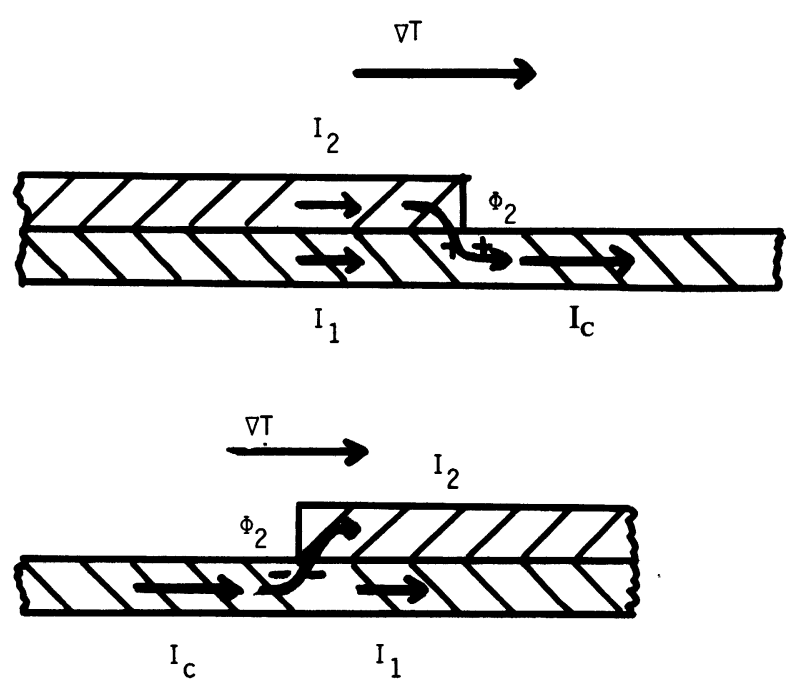

Fig. 2. - Charges électriques induites par effet thermoélectrique sur la frontière du dépôt électrolytique.

[Thermoelectrically induced electrical charges on the outlines of electrolytic deposit.]

Le premier terme des intégrales qui dérive d'un gradient s'annule sur un circuit fermé; le courant $I$ de valeur constante peut être sorti des intégrales. Il en résulte pour la valeur du courant :

$$
\begin{aligned}
& \Delta V=\int_{\mathrm{s}} \frac{I_{1}^{\prime \prime}}{\sigma_{1} e_{1}} \mathrm{~d} l+ \\
& \quad+I\left[\int_{\mathrm{c}} \frac{\mathrm{d} l}{\sigma_{1} e_{1}}+\int_{\mathrm{s}} \frac{\mathrm{d} l}{\sigma_{1} e_{1}+\sigma_{2} e_{2}}\right]
\end{aligned}
$$

Soit en posant

$$
R_{\mathrm{c}}=\int_{\mathrm{c}} \frac{l}{\sigma_{1} e_{1}} \mathrm{~d} l \quad \text { et } \quad R=\int \frac{\mathrm{d} l}{\sigma_{1} e_{1}+\sigma_{2} e_{2}}
$$

$R_{\mathrm{c}}$ est la résistance électrique des connexions et $R$ la résistance électrique de la surface d'échange constituée par les résistances des deux feuilles conductrices assemblées en parallèle :

$$
\Delta V=\varepsilon_{\mathrm{s}}+I\left(R+R_{\mathrm{c}}\right) \text { avec } \varepsilon_{\mathrm{s}}=\int_{\mathrm{s}} \frac{I_{1}^{\prime \prime}}{\sigma_{1} e_{1}} \mathrm{~d} l .
$$

La tension $\Delta V$ induite dans le circuit thermoélectrique est due à des sources thermoélectriques d'ampli- tudes proportionnelles aux courants de densité $I_{1}^{\prime \prime}$ localisées sur la surface d'échange du thermocouple. La tension induite peut être calculée par intégration des tensions élémentaires générées sur la surface d'échange. Dans le cas particulier du thermoélément monodimensionnel représenté figure 1 :

$$
\varepsilon_{\mathrm{s}}=\frac{\sigma_{2} e_{2}}{\sigma_{1} e_{1}+\sigma_{2} e_{2}}\left(\alpha_{2}-\alpha_{1}\right) \Delta T
$$

$\Delta T$ étant la différence de température entre les deux isothermes confondue avec les frontières du capteur.

\subsection{EFFETS THERMoÉLECTRIQUES LOCALISÉS SUR LES} FRONTIÈRES DU THERMOCOUPLE. - Dans la partie précédente, nous avons montré que la tension mesurée dans le circuit noté 1 est représentative des seules chutes ohmiques provoquées par la circulation des courants d'origine thermoélectrique (puisque les tensions d'origine thermoélectrique s'annulent sur un circuit fermé).

La tension globale apparaissant dans un circuit complexe est obtenue par intégration de la quantité $I_{1}^{\prime \prime} / \sigma_{1} e_{1}$ sur toute la surface du circuit. Le même résultat aurait pu être obtenu en considérant comme terme source les charges électriques localisées sur la surface de séparation des conducteurs superposés apparaissant lorsque les courants induits passent du conducteur 1 dans le conducteur 2 et inversement. Ces charges sont localisées aux endroits où le gradient thermique tangentiel a une valeur non nulle. Il y a également apparitions de charges électriques sur le contour du thermoélément supposé soumis à un gradient thermique tangentiel.

Considérons donc un élément de frontière du thermoélément. Soit $I^{\prime}$ et $\phi^{\prime}$ les valeurs des densités par unité de largeur du courant électrique et du flux thermique traversant normalement cet élément de frontière en provenance du dépôt électrolytique. La figure 2 représente une section de la frontière. Il y a continuité des composantes tangentielles des flux thermique et électrique.

$$
I^{\prime}=I_{1 \mathrm{c}} \quad \phi^{\prime}=\phi_{1 \mathrm{c}}
$$

$I_{1 \mathrm{c}}$ et $\phi_{1 \mathrm{c}}$ représentant les densités de flux électrique et thermique dans la connection de sortie constituée par le conducteur noté 1 .

Pour pouvoir exploiter cette condition de passage, il faut utiliser le flux thermique dans l'équation (1) définissant l'état électrique local. En combinant (1) et (2), il vient :

$$
j=\sigma^{\prime} E+\frac{\alpha \sigma^{\prime}}{\lambda} \phi
$$

avec $\sigma^{\prime}=\frac{\sigma}{1+\frac{\alpha^{2} \sigma T}{\lambda}}$ conductivité électrique à flux

thermique nul. 
Partant de cette relation représentative de l'état local d'un conducteur homogène, nous obtenons :

$$
I_{1 \mathrm{c}}=e_{1} \sigma_{1} E_{\mathrm{c}}+\frac{\alpha_{1} e_{1} \sigma_{1}^{\prime}}{\lambda_{1}} \phi_{\mathrm{c}} .
$$

De la même façon, les courants $I_{1}$ et $I_{2}$ provenant du dépôt électrolytique sont définis par la relation :

$$
\begin{aligned}
& I_{1}=\sigma_{1}^{\prime} e_{1} E_{q}^{\prime}+\frac{\alpha_{1} e_{1} \sigma_{1}^{\prime}}{\lambda_{1}} \phi_{1} \\
& I_{2}=\sigma_{2}^{\prime} e_{2} E_{\phi}^{\prime}+\frac{\alpha_{2} e_{2} \sigma_{2}^{\prime}}{\lambda_{2}} \phi_{2}
\end{aligned}
$$

soit au total :

$$
\begin{aligned}
I^{\prime}=I_{1}+I_{2}= & \left(\sigma_{1}^{\prime} e_{1}+\sigma_{2}^{\prime} e_{2}\right) E_{q}^{\prime}+ \\
& +\frac{\alpha_{1} e_{1} \sigma_{1}^{\prime}}{\lambda_{1}} \phi_{1}+\frac{\alpha_{2} e_{2} \sigma_{2}^{\prime}}{\lambda_{2}} \phi_{2}
\end{aligned}
$$

et la condition de passage $I^{\prime}=I_{1 \mathrm{c}}$ conduit à la relation :

$$
\begin{aligned}
\left(\sigma_{1}^{\prime} e_{1}+\sigma_{2}^{\prime} e_{2}\right) & E^{\prime}-\sigma_{1} e_{1} E_{\mathrm{c}}= \\
= & -\left(\frac{\alpha_{2} e_{2} \sigma_{2}^{\prime}}{\lambda_{2}}-\frac{\alpha_{1} e_{1} \sigma_{1}^{\prime}}{\lambda_{1}}\right) \phi_{2} .
\end{aligned}
$$

Cette relation peut encore être simplifiée lorsque la loi de Wiedman Franz est vérifiée $\left(\lambda_{1} / \sigma_{1}^{\prime}=\lambda_{2} / \sigma_{2}^{\prime}\right)$

$$
\begin{aligned}
\left(\sigma_{1}^{\prime} e_{1}+\sigma_{2}^{\prime} e_{2}\right) E^{\prime}-\sigma_{1} e_{1} & E_{\mathrm{c}}= \\
& =-\left(\alpha_{2} e_{2}-\alpha_{1} e_{1}\right) \frac{\sigma_{2}^{\prime}}{\lambda_{2}} \phi_{2} .
\end{aligned}
$$

Le premier membre égal à la différence entre les intensités purement électriques $\left(\sigma_{1}^{\prime} e_{1}+\sigma_{2}^{\prime} e_{2}\right) E$ et $e_{1} \sigma_{1} E_{1}$ représente une charge électrique localisée sur la frontière du dépôt électrolytique. Il y a donc apparition de charges sur les frontières du dépôt électrolytique. Ces charges générées par effet thermoélectrique induisent une tension dans le circuit thermoélectrique. Le terme, source d'origine thermoélectrique, est entretenu par le passage du flux $\phi_{2}$ du conducteur 1 dans le conducteur 2 .

Globalement, l'effet thermoélectrique sur les frontières du thermocouple est matérialisé par une densité linéique de charges proportionnelles à la valeur du gradient thermique tangentiel sur la frontière du thermocouple. Il en résulte un déséquilibre électrique et la circulation de courants induits dans le circuit constantan. Les chutes ohmiques provoquent l'apparition d'une tension $\varepsilon_{\mathrm{f}}$ qui s'ajoute à la tension $\varepsilon_{\mathrm{s}}$ calculée précédemment.

Des travaux sont actuellement développés dans notre laboratoire pour caractériser l'effet thermoélectrique localisé sur les frontières des dépôts électrolytiques.
Le circuit équivalent correspondant à cette caractéristique est représenté figure 3. Il est constitué par un générateur de tension idéal de f.é.m. à vide proportionnelle à la différence de température imposée sur la surface d'échange en série avec une résistance interne égale à $R+R_{\mathrm{c}}$. La tension $\Delta V$ observable aux bornes du conducteur noté 1 à vide est une mesure de la somme des effets thermoélectriques dus aux charges électriques et au passage des courants d'origine thermoélectrique (Fig. 1). Elle est représentative d'effets distribués sur toute la surface d'échange du thermocouple de surface. La tension $\Delta V$ dépend des détails de la distribution spatiale des variations de température de surface. Elle prend une valeur maximale lorsque la distribution de température est antisymétrique par rapport aux coordonnées d'espace [1].
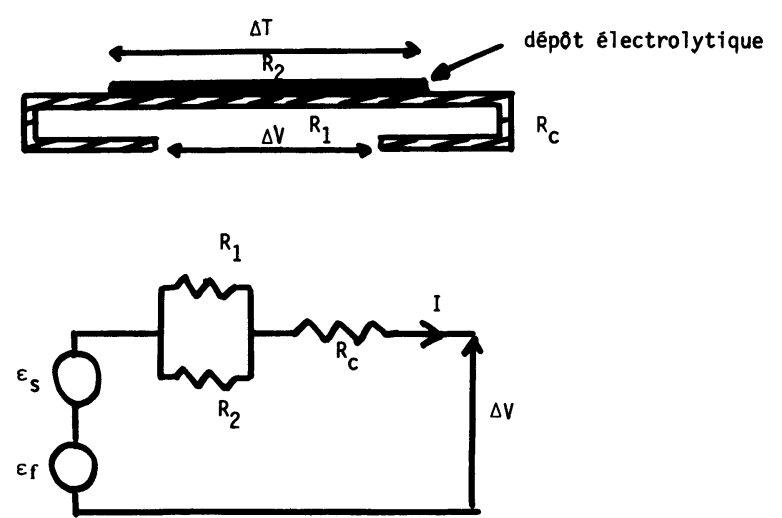

Fig. 3. - Schéma électrique équivalent à un thermocouple de surface.

[Equivalent network to the electrical output of a surface thermocouple.]

$\mathrm{La}$ résistance interne du thermocouple de surface $R+R_{\mathrm{c}}$ est représentative de la résistance des conducteurs ayant une conductivité variable avec la température. La mesure de la résistance interne permet de déterminer la température moyenne du thermocouple. Lorsque la résistance électrique des connexions ne dépend pas de la température, les variations de résistance interne sont représentatives des variations de température moyenne de la surface d'échange du thermocouple. Ces conditions sont réalisées dans les dispositifs étudiés dans notre laboratoire puisque les connexions de sortie sont en constantan. La variation relative de résistance interne avec la température est de l'ordre de $3 \times 10^{-3} /{ }^{\circ} \mathrm{C}$.

Pour augmenter les valeurs de la tension à vide et de la résistance interne, il est intéressant d'assembler les thermocouples de surface en série. Dans les fluxmètres réalisés, 1250 thermocouples sont assemblés en série.

2. Description des cellules fluxmétriques élémentaires réalisées. - 2.1 LES CELLULES FLUXMÉTRIQUES CLASSIQUES À MESURE DIFFÉRENTIELLE DE TEMPÉRATURE 
DE PART ET D'AUTRE D'UNE PAROI AUXILIAIRE. Lorsqu'un flux de chaleur traverse une paroi solide, il apparaît entre les surfaces limites une différence de température proportionnelle à l'amplitude du flux thermique. La connaissance de la chute de température permet de mesurer le flux thermique en régime permanent lorsque la résistance thermique de la paroi auxiliaire a été préalablement mesurée. Les premiers fluxmètres ont été réalisés sur ce principe en $1924[4,5]$ suivant le schéma de principe représenté figure 4. Une feuille de caoutchouc de $2 \mathrm{~mm}$ d'épaisseur et de $60 \times 600 \mathrm{~mm}^{2}$ de surface est utilisée čomme
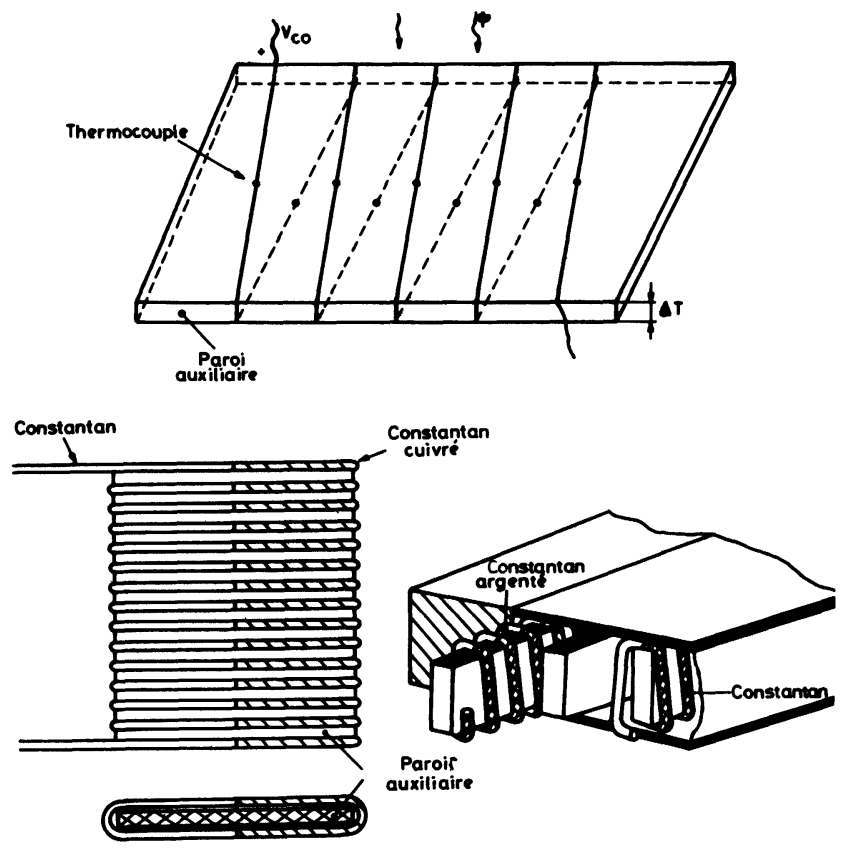

Fig. 4. - Schéma de principe d'un fluxmètre à paroi auxiliaire. Réalisations industrielles.

[Heat fluxmeter with auxiliar wall-industrial realisations.]

paroi auxiliaire. Une thermopile constituée par 100 thermocouples montés en série est utilisée pour mesurer la différence de température entre les surfaces externes de la paroi auxiliaire. Les résistances thermiques des fils constituant la pile thermoélectrique sont tels que la perturbation du champ thermique dans la paroi auxiliaire peut être négligée. Le système de mesure ainsi réalisé est protégé par une couche de caoutchouc vulcanisé de sorte que la pile thermoélectrique est totalement emprisonnée dans le caoutchouc, les seuls fils visibles étant les connexions des liaisons extérieures. L'épaisseur totale du capteur réalisé est de $3 \mathrm{~mm}$.

Les piles thermoélectriques ont également été utilisées dans le domaine des mesures calorimétriques [6]. Les problèmes de mesure des flux de chaleur par pile thermoélectrique ont été analysés en détail. L'information électrique n'est proportionnelle au flux de chaleur inducteur que lorsque l'intégration de thermocouples est suffisante.
Plusieurs types de fluxmètres à paroi auxiliaire ont été réalisés depuis. Le circuit thermoélectrique est constitué par une succession de tronçons de fils de nature différente connectés en série. Les soudures entre les métaux sont réalisées alternativement sur chaque face de la paroi auxiliaire de façon à former un réseau de contacts équidistants $[7,8,9]$. La sensibilité de ces dispositifs dépend de la résistance thermique de la paroi auxiliaire. Elle est généralement comprise entre 100 et $800 \mu \mathrm{V} / \mathrm{W}$ pour des fluxmètres de surface égale à $25 \times 25 \mathrm{~cm}^{2}$, soit entre 62,5 et $500 \mathrm{mV} / \mathrm{W} / \mathrm{cm}^{2}$.

Une innovation introduite dès 1942 a permis de simplifier la méthode de fabrication des fluxmètres à paroi auxiliaire dans la mesure où elle supprime le problème de la réalisation des soudures. La pile thermoélectrique est constituée d'un fil homogène enroulé sur la paroi auxiliaire (Fig. 4). La moitié de l'enroulement est nue, l'autre moitié est recouverte d'un dépôt électrolytique. Les thermocouples sont localisés sur les tronçons recouverts de dépôts électrolytiques. Cette méthode de fabrication a conduit à des réalisations industrielles (Fig. 4).

2.2 Cellules fluXmétriQUes À INTÉgRation DE THERMOÉLÉMENTS. - Le thermocouple de surface étudié précédemment a été utilisé pour réaliser plusieurs types dẹ cellules fluxmétriques. Les thermoéléments sont réalisés en déposant par voie électrolytique une couche de cuivre d'épaisseur voisine de $1 \mu$ sur une feuille de constantan de $25 \mu$ d'épaisseur.

Les cellules fluxmétriques réalisées à partir de thermocouples de surface peuvent être divisées en deux classes. Les éléments de la première classe ou à paroi auxiliaire ont un fonctionnement classique. Ils fournissent une information électrique proportionnelle à la différence de température aux bornes d'une paroi auxiliaire.

Les thermofluxmètres de la deuxième classe ont le grand avantage de permettre la mesure d'un flux dans un plan de section droite (sans nécessité d'une paroi auxiliaire). Le passage de la chaleur à travers une feuille de constantan recouverte de dépôts électrolytiques de cuivre est perturbée au voisinage des frontières de chacun des thermoéléments [10]. Il en résulte une distorsion des lignes de flux et des effets thermoélectriques localisés sur les frontières des thermocouples.

\subsection{Cellules ClassiQues À paroi auXillaire. -} Partant d'une feuille conductrice recouverte d'un dépôt électrolytique de nature différente, il est possible de réaliser une cellule fluxmétrique à paroi auxiliaire (Fig. 5). La paroi auxiliaire étant placée dans le capteur de gradient thermique replié sur lui-même, la tension électrique détectée est proportionnelle à la différence de température aux bornes de la paroi auxiliaire. L'information obtenue est alors proportionnelle au flux thermique à mesurer lorsque le 
temps caractéristique des variations de flux est grand vis-à-vis de la constante de temps fondamentale de la paroi auxiliaire.

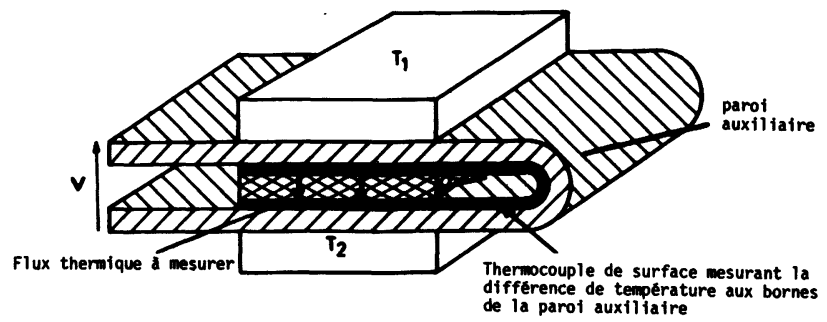

Fig. 5. - Cellule fluxmétrique à effet thermoélectrique distribué sur la surface du thermoélément.

[Thermoelectrical interactions distributed on the area of a heat fluxmeter cell with auxiliar wall.]

\subsection{Cellules À efFet THERMó́leCtriQue Distribút} SUR LES FRONTIÈRES DU THERMOÉLÉMENT. - Il n'est pas indispensable d'utiliser une paroi auxiliaire pour réaliser une cellule fluxmétrique. Nous avons observé qu'un thermoélément à contour dissymétrique (dont une vue de dessus est représentée figure 6) fournit une information électrique proportionnelle au flux thermique traversant la surface du thermoélément.

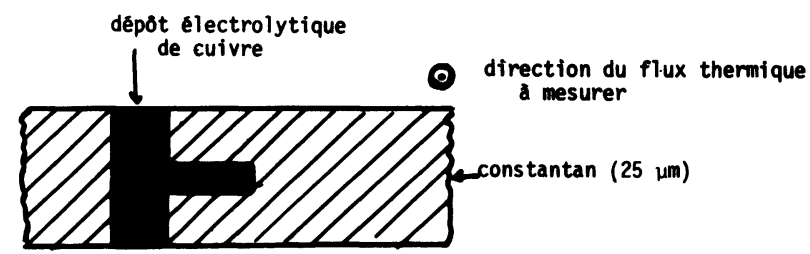

Fig. 6. - Cellule fluxmétrique à contour dissymétrique (vue de dessus). Le plan de la cellule est disposé perpendiculairement au trajet de la chaleur.

[Heat fluxmeter cell with dissymetrical outline.]

Le cuivre électrolytique constituant les thermoéléments étant thermiquement beaucoup plus conducteur que le constantan, il y a déviation des lignes de flux thermique au voisinage des frontières de chaque thermoélément (phénomène de constriction). Les résultats actuellement disponibles [11] peuvent être utilisés pour représenter les distorsions des lignes de champ au voisinage des frontières de chaque thermoélément. Il en résulte l'apparition d'une densité linéique de charges électriques sur la frontière de la région recouverte d'un dépôt électrolytique. Lorsque le contour du thermoélément est symétrique, le champ électrique créé par ces charges électriques suivant la direction principale du thermoélément est nulle et le système reste en équilibre électrique. Il n'en est pas de même lorsque le contour est dissymétrique. Les quantités des charges induites sur chacune des frontières sont inégales. Il en résulte un déséquilibre électrique et l'apparition de courants électriques dans le circuit constantan. La tension observée aux bornes du circuit constantan représentative des seules chutes ohmiques dues à la circulation des courants induits est proportionnelle au degré de dissymétrie du contour de la cellule fluxmétrique.

\subsection{ADDITION DES INTERACTIONS EN SURFACE ET SUR} LES FRONTİ̀RES DES THERMOÉLÉmENTS. - De façon plus générale, il est possible de réaliser une cellule fluxmétrique en créant une dissymétrie dans le plan d'un thermocouple de surface. Dans la cellule représentée figure 7, la dissymétrie est réalisée par une couche conductrice recouvrant partiellement la surface du thermoélément. Il y a dissymétrie du champ thermique à la fois sur la surface et sur les frontières du thermoélément.

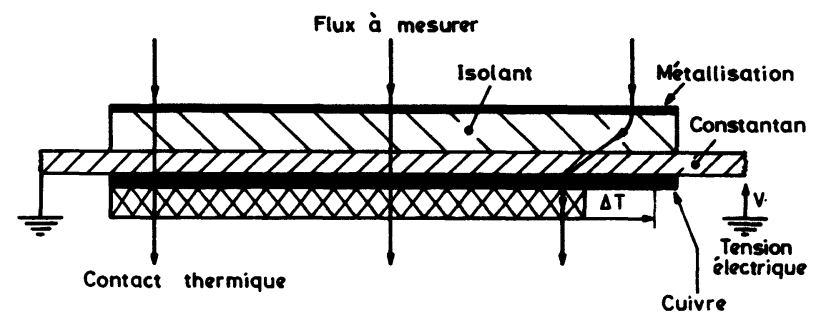

Fig. 7. - Thermoélément à effets thermoélectriques distribué sur la surface d'échange et localisé sur les frontières du thermocouple.

[Thermoelectrical interaction distributed on the aera and located on the limit of a heat fluxmeter cell.]

3. Intégration des thermoéléments. - Le coefficient de sensibilité des thermoéléments est faible puisque les cellules fluxmétriques n'utilisent que la distorsion des lignes de flux pour générer l'information électrique. Pour augmenter la sensibilité, les thermoéléments sont connectés en série. La figure 8 représente une rangée de cellules à contour dissymétrique soumises au même flux thermique. Ce mode de connexion des thermoéléments en série permet d'effectuer la somme des tensions induites par chaque cellule fluxmétrique et d'obtenir ainsi une information électrique proportionnelle au flux de chaleur à mesurer. Les thermofluxmètres ont l'avantage d'être réalisables par mise en ouvre de méthodes classiques de fabrication des circuits imprimés. Partant d'un laminé de constantan d'épaisseur $25 \mu$ sur support kapton (disponible dans le commerce), nous avons obtenu par gravure chimique un circuit de $7 \mathrm{~m}$ de longueur et de $1,8 \mathrm{~mm}$ de largeur replié sur un carré de $12 \times 12 \mathrm{~cm}^{2}$. Le circuit ainsi réalisé est revêtu d'une résine photosensible aux endroits à protéger du cuivrage. 1250 cellules à contour dissymétrique ont pu être disposées sur la surface de mesure du fluxmètre. Les thermoéléments ainsi réalisés sont protégés par une résine thermodurcissable.

Placées entre échangeurs à température imposée, l'ensemble des cellules élémentaires étudiées précédemment fournissent une information électrique proportionnelle au flux thermique traversant le plan de 

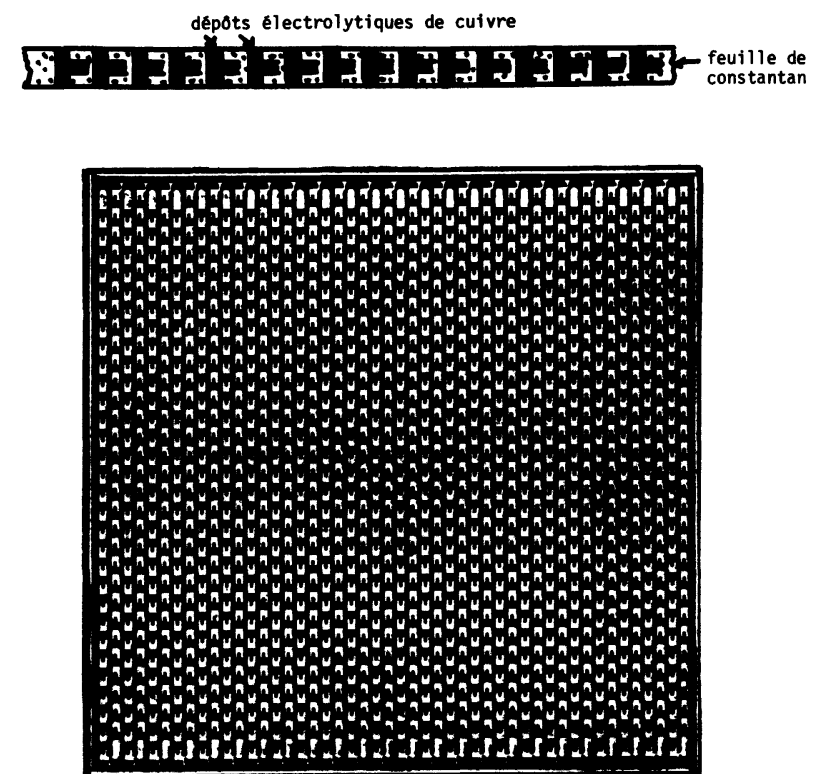

Fig. 8. - a) Rangée fluxmétrique à thermoéléments dissymétriques; $b$ ) Surface de mesure du thermofluxmètre à intégration de thermoéléments dissymétriques.

[a) Row of heat fluxmeter cells with dissymetrical contour. b) Corresponding measurement area.]

section droite. Cet ensemble ne constitue pas pour cela un fluxmètre thermique. Un fluxmètre est un capteur d'usage plus général qui doit fournir une information électrique proportionnelle au flux thermique, quelles que soient les conditions limites imposées sur les surfaces externes du capteur. Les distorsions des lignes de flux doivent être indépendantes de la nature des matériaux disposés de part et d'autre du fluxmètre. Pour réaliser cette condition en pratique, une feuille adhésive mince $(e \sim 50 \mu \mathrm{m})$ en aluminium a été appliquée sur la surface de mesure $\mathrm{du}$ fluxmètre. La sensibilité des capteurs réalisés est de l'ordre de $600 \mu \mathrm{V} / \mathrm{W} \mathrm{dm}^{2}$ [12].

4. Mesure simultanée de flux et de température. Pour mesurer les variations de résistance indépendamment de la tension à vide présente entre les connexions de sortie, le fluxmètre est placé dans l'un des bras d'un pont de Wheastone alimenté en courant alternatif. Dans ces conditions, l'impédance apparente du thermocouple est représentée par sa résistance interne et les variations de température moyenne peuvent être mesurées indépendamment des différences de température présentes sur la surface d'échange. Le montage de la figure 9 représente 'e fluxmètre placé dans la branche d'un pont de Wheatsone. Dans ces conditions, le fluxmètre fonctionne en circuit fermé et induit une tension proportionnelle au flux thermique dans la diagonale $A B$ $\mathrm{du}$ pont de Wheatsone se superposant à la tension alternative $V_{0}$ alimentant le pont $(f=1000 \mathrm{~Hz})$.

Pour séparer la composante proportionnelle au flux de chaleur de la composante alternative la

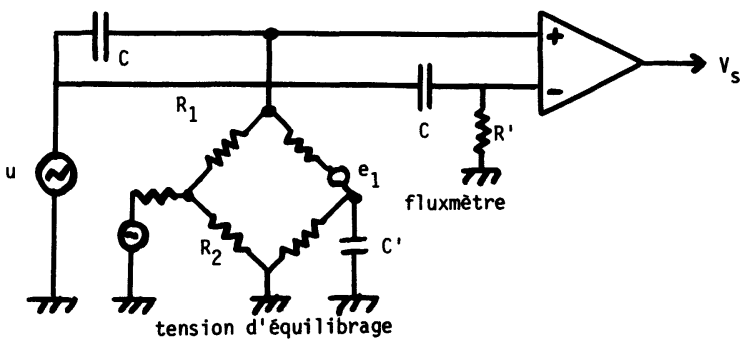

Fig. 9. - Pont de Wheastone à équilibrage automatique.

[Automatically balanced Wheaston bridge.]

tension aux bornes de la diagonale $\mathrm{AB}$ est appliquée sur l'entrée positive d'un amplificateur différentiel :

$$
V_{+}=V_{0} \sin \omega t+\frac{\left(R_{1}+R_{2}\right)\left(e_{1}\right)}{r_{1}+r_{2}+R_{1}+R_{2}}
$$

$e_{1}$ étant la tension à vide mesurée aux bornes du fluxmètre,

$r_{1}$ la résistance interne du fluxmètre.

Le signal d'alimentation $V_{0}$ est appliqué sur l'entrée négative de l'amplificateur :

$$
\begin{aligned}
& V_{-}=V_{0} \sin t \omega t \\
& V_{1}=V_{+}-V_{-}=\frac{R_{1}+R_{2}}{R_{1}+R_{2}+r_{1}+r_{2}} e_{1} .
\end{aligned}
$$

La sortie de l'amplificateur différentiel est proportionnelle à la tension $e_{1}$ à mesurer.

Pour effectuer une mesure différentielle de flux thermique, il est possible d'utiliser le même montage en remplaçant la résistance $r_{1}$ par un second fluxmètre de résistance interne égale à $r_{2}$.

Pour mesurer la résistance interne $r_{2}$ qui fournit une information représentative de la température de la surface de mesure, nous équilibrons le pont en alternatif $(f \sim 1000 \mathrm{~Hz})$. Cette fonction est réalisée par une chaîne d'équilibrage automatique. Le dispositif met en ouvre une boucle de contre-réaction numérique générant à chaque instant un signal analogique rétablissant l'équilibre du pont. La constante de temps du système d'équilibrage est voisine de $40 \mathrm{~ms}$. L'information numérique représentant le déséquilibre du pont est obtenue par traitement du signal de déséquilibre. Le dispositif présente l'avantage par rapport aux systèmes classiques qui exploitent directement la tension de déséquilibre de fournir directement une information rigoureusement proportionnelle aux variations de résistance à mesurer quelles que soient leurs amplitudes. Les écarts par rapport à la linéarité sont inférieures à $1 \%$ dans la gamme d'utilisation. Le module électronique permet de mesurer la température du fluxmètre en régime variable [13].

4.1 Applications. - Le thermofluxmètre réalisé diffère de par son principe des autres capteurs du même type actuellement disponibles puisque l'infor- 
mation électrique n'est pas proportionnelle à la différence de température aux bornes d'une paroi auxiliaire. L'information électrique n'est pas proportionnelle à la valeur instantanée d'une différence de température. Il en résulte une information totalement différente en régime transitoire puisque l'information fournie par un fluxmètre à paroi auxiliaire en régime variable résulte d'une intégration temporelle des variations de flux thermique.

Compte tenu de ces considérations, le capteur réalisé est particulièrement utile en régime transitoire. Nous avons pu l'utiliser pour évaluer la constante de temps fondamentale d'une paroi par analyse du flux transmis en régime impulsionnel $[14,15]$.

D'autres applications particulièrement intéressantes résultent de la possibilité de mesurer simultanément flux et variations de température en régime variable. Disposant de ces variations, un traitement simple permet de calculer le flux d'entropie et d'évaluer l'augmentation d'entropie par dissipation interne dans les transformations cycliques [16].

5. Conclusion. - Partant des équations phénoménologiques représentatives du couplage thermoélectrique dans un conducteur homogène, nous avons pu décrire les propriétés des thermocouples de sur- face. La tension mesurée aux bornes de ces dispositifs est induite par la composante tangentielle du gradient thermique. L'effet thermoélectrique est distribué sur la surface d'échange et les frontières du thermoélément. Un développement intéressant aussi bien du point de vue théorique que du point de vue pratique consisterait à étendre l'étude aux thermoéléments à trois dimensions réalisables par gravure, puis cuivrage électrolytique.

Disposant de thermocouples de surface, il est possible de réáliser divers types de cellules fluxmétriques. Les thermofluxmètres à intégration de thermocouples présentent par rapport aux dispositifs antérieurs l'avantage de fournir une mesure de flux dans un plan de section droite (sans la nécessité d'une paroi auxiliaire). L'information électrique est générée par la distorsion des lignes de flux thermiques au voisinage des régions cuivrées déposées sur le circuit constantan. Une modélisation de ces phénomènes permettra d'optimiser la sensibilité des thermofluxmètres.

La résistance électrique du circuit constituant le thermofluxmètre dépend de la température. La mesure de cette résistance en régime sinusoïdal permet d'éliminer les effets thermoélectriques et conduit à la mesure simultanée du flux thermique et de la température moyenne dans un plan de section droite.

\section{Bibliographie}

[1] Théry, P., Pauquet, J., J. Phys. E., Sci. Instrum. 13 323-327.

[2] Théry, P., Duthoit, B., Pauquet, J., Revue Phys. Appl. 15 (1980) 741-747.

[3] Théry, P., Maréchal, J. C., J. Phys. E. Instrum. 13 (1980) 859-865.

[4] Sнмid, E., Arch. Wärmewirt 1924, a no 1, p. 9.

[5] JACOB, M., Heat Transfer (John Wiley Son, Edit.) 1957, 194-195.

[6] Calvet, E., Prat, H., Microcalorimétrie (Edit. Masson) 1956, chap. 2, p. 21.

[7] KLARSFELD, S., International Standart 150, 2581 (1975) (E).

[8] Maréchal, J. C., IIF Nantes (1957).

[9] Devisme, Maréchal, J. C., Ann. ITBTP 165 54-70.

[10] Bardon, J. P., communication privée.

[11] Bardon, J. P., Rev. Gén. Therm. Fr. 170 (1976) 121-135.
[12] Cassagne, B., Sté Française des Thermiciens, journée du 10 mars 1981.

[13] LeclercQ, D., Théry, P., Journées Ind. de la Sté Française de Physique, Toulouse, mars 1980.

[14] Kougbeadjo, R. A., Thèse Docteur Ingénieur, Janvier 1981, Univ. de Lille 1 «Identification de la diffusivité thermique par traitement du transfert de chaleur à travers une paroi homogène en régimes impulsionnel et pseudo-aléatoire. Application des méthodes de corrélation en thermocinétique ".

[15] Duthoit, B., Farza, A., Théry, P., « Mesure de la dégradation énergétique dans les processus de stockage et de transfert de la chaleur ", à paraître, Int. J. Heat Mass Transfer, 1982.

[16] Kougbeadjo, R. A., Thery, P., J. Appl. Phys. (April 1982 à paraître). 